\title{
Polibotánica
}

ISSN electrónico: 2395-9525

POLIBETÁNICA polibotanica@gmail.com Instituto Politécnico Nacional México http:www.polibotanica.mx

\section{DESCOMPOSICIÓN DE LA MADERA DE ROBLE (Quercus spp.) IN VIVO E IN VITRO}

\section{IN VIVO AND IN VITRO WOOD DECAY OF OAK (Quercus spp.)}

Carranza-V., J.; J.F. Di Stéfano-G., W. Marín-M., y M. Mata-H.

DESCOMPOSICIÓN DE LA MADERA DE ROBLE (Quercus spp.) IN VIVO E IN VITRO. IN VIVO AND IN VITRO WOOD DECAY OF OAK (Quercus spp.).

POLIB RÁNICA

Instituto Politécnico Nacional
Núm. 47: 59-76 México. Enero 2019

DOI: $10.18387 /$ polibotanica.47.5 
DESCOMPOSICIÓN DE LA MADERA DE ROBLE (Quercus spp.) IN VIVO E IN VITRO

\title{
IN VIVO AND IN VITRO WOOD DECAY OF OAK (Quercus spp.)
}

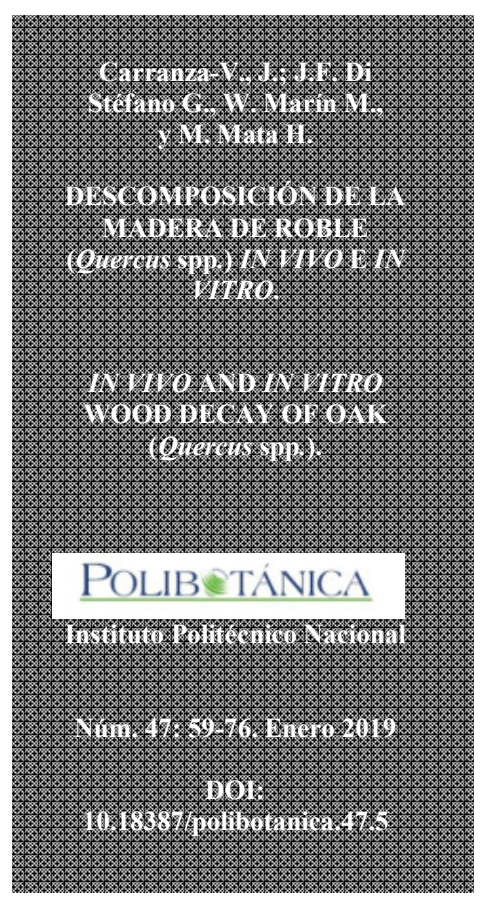

\author{
J. Carranza-V./ julieta.carranza@ucr.ac.cr; carranza.julieta@gmail.com \\ Escuela de Biología, Universidad de Costa Rica \\ Laboratorio de Recursos Forestales, Instituto de Investigaciones en Ingeniería, \\ Universidad de Costa Rica, San Pedro, Costa Rica \\ J.F. Di Stéfano-G. \\ Escuela de Biología, Universidad de Costa Rica, San Pedro, Costa Rica \\ W. Marín-M. \\ M. Mata-H. \\ Escuela de Biología, Universidad de Costa Rica \\ Laboratorio de Recursos Forestales, Instituto de Investigaciones en Ingeniería, \\ Universidad de Costa Rica, San Pedro, Costa Rica
}

RESUMEN: La descomposición de los restos de madera en los bosques ocasiona un flujo considerable de carbono. Las tasas de descomposición varían en zonas templadas y tropicales y de acuerdo al tipo de madera y a los hongos que están involucrados en este proceso. Varios estudios relacionados con la descomposición de la madera de roble han sido llevados a cabo en zonas templadas, pero éstos son escasos o inexistentes en bosques neotropicales de altura. Esta investigación se llevó a cabo para determinar el avance en los niveles de podredumbre (1-4) de troncos y tocones de roble en un bosque primario y en uno secundario en Costa Rica, en diferentes estaciones a lo largo de cinco años, así como para observar el proceso de descomposición en muestras de madera de roble, expuestas a hongos seleccionados en condiciones de laboratorio. Los niveles de podredumbre de los troncos y tocones en los bosques se mantuvieron -en su mayoríaen el nivel 1 durante todo el estudio. Para las pruebas de podredumbre se seleccionaron tres hongos comunes en los troncos de roble: Xylobolus subpileatus, Hymenochaete rubiginosa y Trametes versicolor, y se utilizaron bloques de madera provenientes de ramas de árboles de la zona de estudio. Se montaron 25 cámaras de podredumbre para cada hongo, y los bloques de madera se removieron a los cuatro u ocho meses para determinar las pérdidas de peso. Se obtuvo una gran variación en los porcentajes de pérdidas de peso (2.08-51.06\%), en todos los tratamientos. Con base en los resultados obtenidos, se discute la posible duración de la descomposición total de la madera de roble en estos bosques neotropicales y su aporte de carbono al ecosistema.

Palabras clave: hongos, descomposición, madera, roble, bosques montanos neotropicales, Xylobolus subpileatus, Hymenochaete rubiginosa, Trametes versicolor, cámaras de podredumbre, Costa Rica.

ABSTRACT: Wood decomposition produces a considerable flow of carbon in the ecosystem, but decomposition rates have been observed to change in tropical areas compared with temperate ones, and are affected by the type of wood and the fungal community involved in the process. Several studies have been carried out with oak wood decomposition in temperate zones but they are scarce or lacking in highland tropical areas. The aim of this study was to determine the degree of decay (1-4) of logs and stumps in two neotropical montane forests in Costa Rica, and to observe the 
decomposition of oak wood samples exposed to selected fungi under laboratory conditions. The most common decay category in the logs and stumps was 1, and it remained that way during the whole study. Xylobolus subpileatus, Hymenochaete rubiginosa, and Trametes versicolor were selected for the decay studies. Twenty-five decay chambers with oak wood samples were inoculated with each fungus. After 4 or 8 months of exposure, wood samples were removed to determine weight loss. Considerable variation was observed in weight loss (2.08-51.06\%), depending on the fungi selected. In light of our results, the duration of the oak decay process in these neotropical montane forests is discussed, as well as its carbon contribution to the ecosystem.

Key words: fungi, wood decomposition, wood, oak, neotropical montane forests, Xylobolus subpileatus, Hymenochaete rubiginosa, Trametes versicolor, decay chambers, Costa Rica.

\section{INTRODUCCIÓN}

Los restos de madera en los bosques (troncos y ramas) juegan un papel muy importante en la estructura y funcionamiento de los ecosistemas, principalmente en los ciclos de carbono y nutrientes. Las tasas de descomposición de estos materiales varían a escala temporal y espacial, de acuerdo con el tipo de bosque, la especie de la planta y del diámetro del sustrato (Freschet et al., 2012; Grove et al., 2011).

Trabajos realizados en zonas templadas y bosques tropicales han comunicado tasas de descomposición de 0.15 a $0.45 \mathrm{~g} \mathrm{~g}^{-1}$ y de 0.11 a $1.23 \mathrm{~g} \mathrm{~g}^{-1}$ por año ${ }^{-1}$, respectivamente, lo cual ocasiona un flujo considerable de carbono, especialmente en zonas tropicales (Boddy \& Swift, 1984; Chambers et al., 2000; Keller et al., 2004; Ostrogović et al., 2015).

Los hongos de podredumbre son cruciales en estos procesos de descomposición ya que están íntimamente involucrados en la toma y reciclaje de los nutrientes minerales, para devolverlos al ecosistema. Estos organismos invaden sustratos que difieren en tamaño, en la densidad de su madera, en los niveles de podredumbre presentes, en su composición química y en su contenido de humedad. Todos los causantes de podredumbre, en cierto grado, degradan compuestos lignocelulósicos, y en consecuencia ablandan los tejidos de la madera y la hacen más accesible para ser habitada por aves y pequeños mamíferos, y utilizada por invertebrados, así como por otros grupos de hongos que participan en los procesos de sucesión. Asimismo, se ha demostrado la importancia de la madera en estados avanzados de podredumbre en el suelo no sólo para el establecimiento de asociaciones micorrízicas con plántulas, sino también porque los residuos de madera descompuesta actúan como sumideros de humedad para el mantenimiento de hongos micorrízicos en bosques estacionales secos (Grove et al., 2011; Huhndorf et al., 2004; Kubartová et al., 2012; Mueller et al., 2006; Pyle \& Brown, 1999).

Muchas variables ambientales influyen en la diversidad de estos organismos en los bosques, entre ellas el microclima, el sustrato, la edad del hospedero, la cubierta boscosa, el tiempo de perturbación, la densidad de cobertura, y la abundancia de árboles caídos (Lindblad, 2001; Lodge, 1993).

Entre los factores más importantes para el crecimiento de los hongos de la madera, se han mencionado la humedad y el tipo de sustrato (Kubartová et al., 2012; Lisiewska, 1992; Whalley, 1993). Los porcentajes de humedad mínimos necesarios oscilan entre 18-20\%, con óptimos entre 25-55\% (Kirk, 1973; Rodríguez-Barreal, 1998; Scheffer, 1973). Pyle y Brown (1999) observaron que el porcentaje de humedad presente en los grados intermedios de podredumbre parece ser el más favorable para su desarrollo. Los sustratos que presenten densidades y durabilidades naturales bajas (con menor cantidad de extractivos) son los más fácilmente degradados por estos organismos (Rodríguez-Barreal, 1998). 
De acuerdo a la durabilidad natural, las maderas se han clasificado en muy resistentes, resistentes, moderadamente resistentes y en poco o nada resistentes (Guilley et al., 2004; Scheffer, 1973). Una de las maderas más resistentes al ataque por hongos e insectos es la de roble (Quercus L.), dado su alto contenido de taninos y su alta densidad (0.54-0.99 $\mathrm{g} \mathrm{cm}^{-3}$ ) así como el grosor de los anillos, aspectos que le dan gran resistencia y dureza (Guilley et al., 2004; Highley, 1995; Sánchez-Chacón et al., 1994; Scheffer, 1973). La tasa de descomposición de diferentes especies de roble ha sido calculada entre 0.03 a $0.069 \mathrm{~g} \mathrm{~g}^{-1}$ por año ${ }^{-1}$, con un tiempo promedio de 25 años, lo que confirma su alta resistencia (Schowalter et al., 1992; Schowalter et al., 1998).

Dado el poco conocimiento sobre la descomposición de los troncos y tocones de roble en bosques neotropicales, se planteó la presente investigación para determinar los niveles de podredumbre de esta madera en un bosque primario y en uno secundario, en diferentes estaciones a lo largo de cinco años, así como para observar los patrones de descomposición de bloques de madera de roble, expuestos a hongos seleccionados en condiciones de laboratorio.

\section{MÉTODOS}

\section{Sitio de estudio}

Esta investigación se llevó a cabo en un bosque secundario en proceso de regeneración desde hace aprox. 32 años (2 751 m s.n.m., latitud norte de $9^{\circ} 35^{\prime} 44^{\prime \prime}$, longitud oeste de $83^{\circ} 47^{\prime} 49^{\prime \prime}$ ) y en uno maduro o primario (2 577 m s.n.m., latitud norte de $9^{\circ} 35^{\prime} 30^{\prime \prime}$, longitud oeste de $83^{\circ}$ $47^{\prime} 28^{\prime \prime}$ ) ubicados en Costa Rica, cerca de los pueblos de San Gerardo y Jaboncillo, distrito de Copey, cantón de Dota, Provincia de San José, $3 \mathrm{~km}$ al suroeste del kilómetro 80 de la Carretera Interamericana Sur, entre el 2008 y 2012 (fig. 1). La distancia entre ambos bosques es de $6 \mathrm{~km}$. La vegetación del bosque maduro se caracteriza por una dominancia de Quercus copeyensis C.H. Mull. y Quercus costaricensis Liebm. que alcanzan diámetros de un metro o más, y alturas mayores a 30 m (Carranza, DiStéfano, Marín \& Mata, 2018). La vegetación del bosque en regeneración se encuentra dominada por Fuchsia arborescens Sims y Abatia parviflora Ruiz \& Pav. junto con las mismas especies de roble (Muñoz-Rivera, 2009).

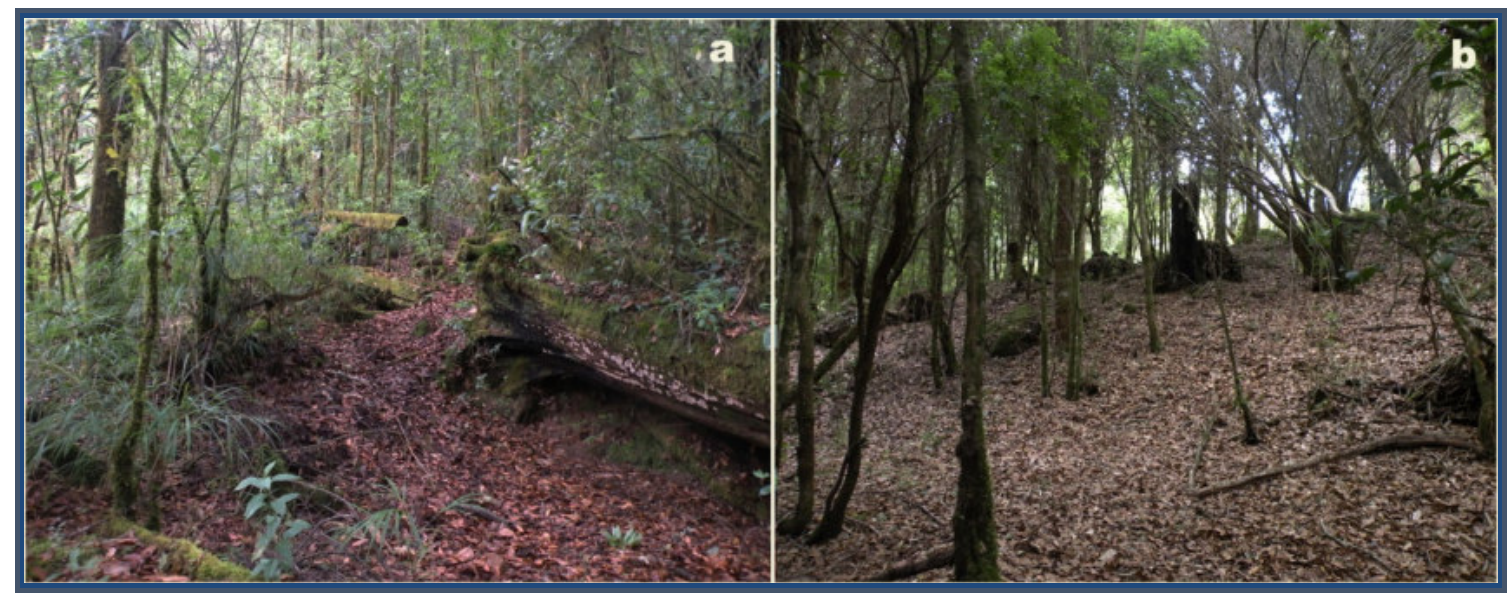

Fig. 1. Bosques montanos neotropicales de Costa Rica. a. Bosque primario o maduro; b. Bosque secundario o en regeneración. 


\section{Datos meteorológicos}

Los datos sobre precipitación, temperatura y humedad relativa cercanos a la zona de estudio (Ojo de Agua y Cerro de la Muerte) para los años 2008-2012, fueron solicitados al Centro de Servicios Estudios Básicos de Ingeniería Hidrología, Instituto Costarricense de Electricidad, San José, Costa Rica (fig. 2). Sin embargo, no se contaba con datos completos de temperatura y humedad relativa para la estación Ojo de Agua para el periodo solicitado. Dado que la zona de estudio se encuentra entre estas dos estaciones, se consideró importante incluir al menos, la información de precipitación para la estación Ojo de Agua, para mostrar las variaciones en la zona.

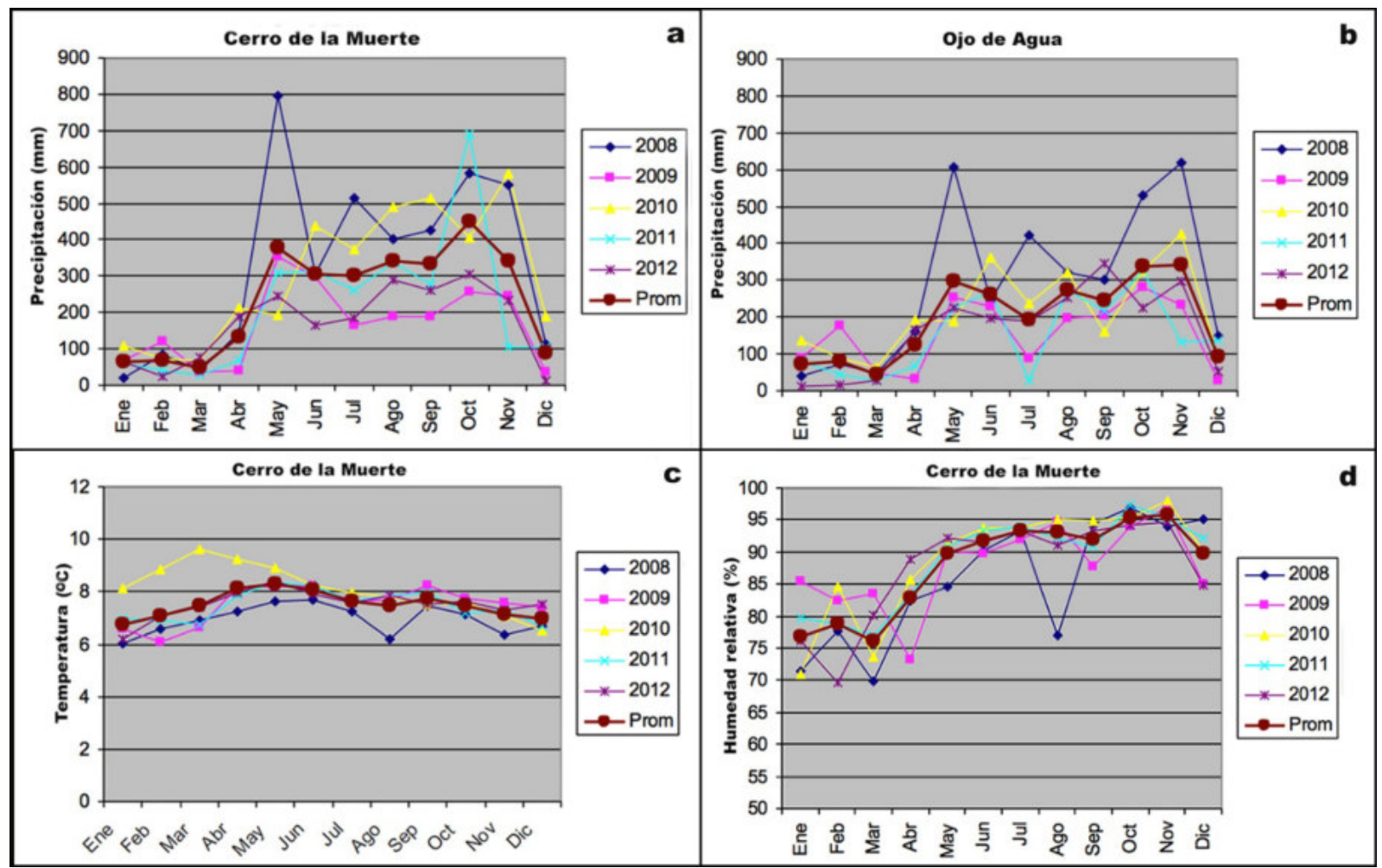

Fig. 2. Datos climatológicos de las estaciones Cerro de la Muerte y Ojo de Agua, Cordillera de Talamanca, para los años 2008-2012. a. Precipitación total mensual por año y promedio; b. Precipitación total mensual por año y promedio; c. Temperatura promedio mensual por año; d. Humedad relativa promedio mensual por año.

\section{Selección de los troncos y determinación del nivel de podredumbre:}

Un total de 41 troncos (30 en bosque primario, 11 en bosque secundario) y 20 tocones (sólo en bosque secundario) fueron seleccionados de forma oportunista (muestreo por conveniencia) en ambos bosques y se marcaron con banderolas y pintura flourescente. No se logró identificarlos a nivel de especie. Los diámetros de los troncos seleccionados oscilaron de 0.2 a $0.68 \mathrm{~m}$, y las longitudes de 1-23.2 m; en el caso de los tocones, los diámetros variaron de 0.28 a $3.94 \mathrm{~m}$, y el alto de 1 a $1.5 \mathrm{~m}$. Los niveles de podredumbre observados al inicio del estudio, variaron de 1 a 4 según la siguiente clasificación de Huhndorf et al. (2004) y Mueller et al. (2004): nivel 1: madera dura sin podredumbre (árbol retiene la corteza o está suelta), nivel 2: madera dura pero con podredumbre, descolorida o no (medianamente podrido, corteza caída, cuchillo penetra $2 \mathrm{~cm}$ dentro de la madera sin ponerle presión), nivel 3: madera suave y 
esponjosa, nivel 4: madera con alto grado de podredumbre (cuchillo penetra sin presión toda la madera, la cual puede ser destruida con los dedos).

En cada muestreo se determinó el avance del nivel de podredumbre de los diferentes troncos y tocones de acuerdo a la metodología supracitada (Huhndorf et al., 2004; Mueller et al., 2004).

\section{Recolecta e identificación de los hongos}

Durante cada visita (cuatro a cinco visitas distribuidas en los diferentes meses del año por cinco años) se recolectaron los cuerpos fructíferos presentes en los troncos y tocones seleccionados para su posterior identificación, de acuerdo a la metodología de Lodge et al. (2004). En cada caso se anotó el número del tronco o tocón donde se encontraban para luego determinar si existía alguna relación entre el tamaño del tronco o tocón y la cantidad de especies observadas.

\section{Muestras de madera para cálculo de humedad, análisis químicos y cámaras de podredumbre}

Se obtuvieron muestras de madera $(10 \mathrm{~cm}$ x $5 \mathrm{~cm})$ de los troncos y tocones seleccionados por medio de un hacha, durante todas las visitas realizadas en las diferentes épocas del año y a través de todo el período de estudio. Dichas muestras se colocaron en bolsas plásticas selladas y se llevaron al laboratorio, para determinar el porcentaje de humedad de acuerdo a Donahue et al. (1983). Todas las muestras fueron pesadas frescas, secadas a $65^{\circ} \mathrm{C}$ en un horno durante una semana hasta peso constante y luego se pesaron de nuevo. Asimismo, se obtuvieron muestras de madera procedentes de ramas de árboles vivos cercanos al bosque secundario, para los análisis químicos y para la preparación de los bloques de las cámaras de podredumbre. Los bloques se prepararon de acuerdo al procedimiento establecido en las normas ASTM D-2017-05 (ASTM, 2005; Sarmiento et al., 2016).

\section{Muestras de suelo para cálculo de humedad}

Durante todas las visitas se recolectaron muestras de suelo para determinar su porcentaje de humedad y determinar las variaciones durante las diferentes épocas del año y a través del periodo de estudio. Las muestras fueron llevadas al laboratorio, pesadas y secadas a $65^{\circ} \mathrm{C}$ en un horno durante una semana. El porcentaje de humedad fue calculado de acuerdo a Donahue et al. (1983).

\section{Evaluación de la capacidad descomponedora de basidiomicetes}

Se seleccionaron dos de los hongos que se encontraban en la mayoría de los troncos de roble en ambos bosques de acuerdo a resultados de estudio previo (Carranza et al., 2018): Hymenochaete rubiginosa (Dicks.) Lév. y Xylobolus subpileatus (Berk. \& M. A. Curtis) Boidin. Asimismo, se incluyó Trametes versicolor L. (Lloyd), por crecer comúnmente en roble y por ser utilizado en estudios de podredumbre a nivel mundial (Guilley et al., 2004; Szewczyk \& Molińska-Glura, 2010), para comparar la habilidad descomponedora de los tres hongos (fig. 3). 


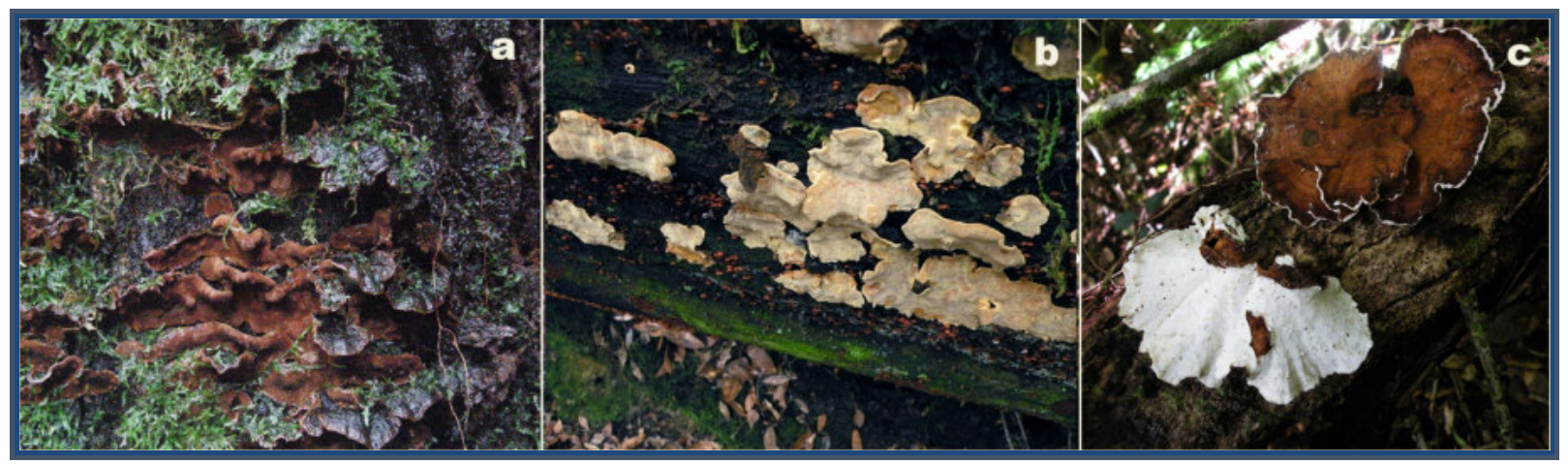

Fig. 3. a-c. Hongos seleccionados para las cámaras de podredumbre. a. Hymenochaete rubiginosa (Dicks.) Lév.; b. Xylobolus subpileatus (Berk. \& M. A. Curtis); c. Trametes versicolor L. (Lloyd).

Se sembraron fragmentos de cuerpos fructíferos o micelios de estos tres hongos seleccionados que se encontraban en los troncos o tocones, en cajas de Petri con extracto de malta agar al $2 \%$ y se incubaron por dos semanas a $26^{\circ} \mathrm{C}$. Se realizaron varias transferencias de los micelios a cajas con el mismo medio hasta obtener cultivos puros, los cuales se utilizaron para inocular las cámaras de podredumbre. El procedimiento seguido se basó en la metodología descrita en Carranza \& Ruiz-Boyer (2001) y Sarmiento et al. (2016).

\section{Preparación de las cámaras de podredumbre}

La preparación de las cámaras se realizó de acuerdo a las normas ASTM D-2017-05 (ASTM, 2005; Sarmiento et al., 2016). Se sembraron inóculos de cada uno de los hongos seleccionados en 25 botellas cuadradas ( 75 en total) con medio de extracto de malta al $2 \%$ y se incubaron a $26^{\circ} \mathrm{C}$. Al cabo de dos semanas, se colocaron tres bloques de madera de roble de $2.5 \times 2.5 \times 1.5$ $\mathrm{cm}$ (previamente pesados y esterilizados) en cada botella inoculada y tres bloques en cada una de 15 botellas con el mismo medio de cultivo, pero sin inocular (testigos). Las cámaras se incubaron a $28^{\circ} \mathrm{C}$ en la oscuridad y se removieron la totalidad de los bloques a los cuatro u ocho meses para el cálculo de pérdida de peso, observación al microscopio y análisis químico.

\section{Cálculo de pérdida de peso de los bloques}

Los bloques removidos se colocaron en un horno de secado a $65 \pm 1^{\circ} \mathrm{C}$ hasta alcanzar un peso constante. La pérdida de biomasa fue determinada con base en el peso seco inicial y final [(peso seco original-peso seco $\mathrm{t}=4 \mathrm{u} 8 \mathrm{meses} /$ peso seco original) $\mathrm{x} 100]$. En el caso de las cámaras con $T$. versicolor, todas las muestras fueron removidas a los cuatro meses, dada su rápida descomposición.

\section{Procesamiento de los bloques para observación de daños anatómicos al microscopio de luz}

Los bloques utilizados para estudios de microscopía de luz fueron procesados en el Laboratorio de Recursos Forestales de la Facultad de Ingeniería, Instituto de Investigaciones en Ingeniería, Universidad de Costa Rica. Se les removió el micelio superficial a los bloques inoculados para descubrir la madera y se colocaron en la solución fijadora FAA (formaldehído, alcohol y ácido acético) para evitar el colapso de las hifas internas para su posterior corte y observación (Carpio Malavassi, 1992).

Las secciones tanto de los bloques de madera sana (testigos) como de los sometidos al ataque de los diferentes hongos, fueron obtenidas por medio de un microtomo de deslizamiento marca Reichert, Austria. Los cortes se realizaron en los planos transversal, longitudinal radial y 
longitudinal tangencial, a un grosor de 30-50 $\mu \mathrm{m}$. Las secciones testigo fueron sumergidas brevemente en una solución concentrada de $\mathrm{HNO}_{3}$ y NaOH para eliminar impurezas debido a la fricción del corte. Las secciones obtenidas de bloques colonizados fueron lavadas en agua destilada para preservar la presencia del hongo. Todas las secciones fueron deshidratadas en series ascendentes de alcohol de $70^{\circ}$ a alcohol absoluto, posteriormente teñidas en una solución de safranina, montadas y selladas con Permount en portaobjetos (Ruzin, 1999).

\section{Análisis químicos a los bloques inoculados}

Muestras de los bloques inoculados y testigos fueron analizadas en el Laboratorio de Celulosa del Instituto de Investigaciones en Ingeniería, Universidad de Costa Rica, para determinar cambios en los contenidos de carbohidratos y lignina durante el proceso de degradación. Los ensayos químicos se realizaron con base en las normas TAPPI (1996) T-264, T-222 y T-211.

\section{Análisis estadísticos}

Se determinaron los promedios y las desviaciones estándar para los datos de pérdidas de peso en los diferentes tratamientos. Se realizó un análisis de regresión lineal para relacionar el número de especies con el tamaño de los troncos (Gomez \& Gomez, 1984).

\section{RESULTADOS}

\section{Niveles de podredumbre de los troncos y tocones en los bosques}

Se observó una gran variación en los niveles de podredumbre tanto en los troncos como en los tocones de ambos bosques desde el inicio hasta el final del estudio. En un mismo tronco o tocón fue común encontrar partes extensas muy duras donde el cuchillo no penetró (nivel 1), con partes más limitadas donde la podredumbre oscilaba de nivel 2 a 3 , y en muy pocos casos, zonas con una podredumbre nivel 4 . Estos estados más avanzados de podredumbre se dieron a los lados, en el centro o en los extremos de los troncos, y en los lados y en el centro de los tocones. Por lo general, los niveles de podredumbre en cada tronco o tocón avanzaron lentamente durante las diferentes estaciones a lo largo de los cinco años de estudio, por lo que ningún tronco o tocón se descompuso completamente.

Del total de troncos y tocones marcados, sólo tres troncos en el bosque primario y uno en el bosque secundario que al inicio del estudio presentaron nivel 1, mostraron al final algunas partes con niveles 3-4.

No fue posible determinar el tiempo de descomposición de los troncos y tocones, ya que no se pudo definir el momento del inicio de la colonización. Sin embargo, en el bosque primario, se había marcado un árbol que cayó en el 2008 y al final de los cinco años mostró solamente una zona pequeña en uno de los extremos con podredumbre nivel 3 , el resto estaba bastante duro y mantenía aún su corteza (nivel 1).

Los niveles de podredumbre donde se encontraban $X$. subpileatus o H. rubiginosa o ambas especies, oscilaron entre 1-4. Cabe mencionar que otras especies de hongos causantes de podredumbre (57 especies), compartían el sustrato con alguna de estas dos especies o con ambas. La presencia de especies de macrohongos descomponedores de madera fue mayor en el bosque primario (41\%) que en el secundario (22\%); sin embargo, ambos bosques compartieron un porcentaje alto $(37 \%)$ del total de las especies causantes de podredumbre presentes en los troncos o tocones (cuadro 1). 
Cuadro 1. Especies de hongos descomponedores de madera asociados a troncos (T) y tocones (To) de roble, en el bosque primario (BP) y secundario (BS), San Gerardo de Dota, San José, Costa Rica. 2008-2012.

\begin{tabular}{|c|c|c|}
\hline Basidiomicetes & Basidiomicetes (cont.) & Ascomicetes \\
\hline $\begin{array}{l}\text { Armillaria puiggarii Speg. (T-To, BS, } \\
\text { podr. 1-2) }\end{array}$ & $\begin{array}{l}\text { Lentinellus ursinus (Fr.) Kühner (To, } \\
\text { BS, podr. 1) }\end{array}$ & $\begin{array}{l}\text { Bisporella citrina (Batsch) Korf } \\
\text { \& S.E. Carp. (T-To, BP-BS, } \\
\text { podr. 1-2) }\end{array}$ \\
\hline Cf. Arrhenia sp.1 (T, BP, podr. 1-2) & $\begin{array}{l}\text { Lentinula boryana (Berk. \& Mont.) } \\
\text { Pegler (T, BP, podr. 1) }\end{array}$ & $\begin{array}{l}\text { Chlorencoelia sp.1 (T-To, BP- } \\
\text { BS, podr. 1-3) }\end{array}$ \\
\hline $\begin{array}{l}\text { Campanophyllum proboscideum (Fr.) } \\
\text { Cifuentes \& R.H. Petersen (T-To, BP- } \\
\text { BS, podr. 1) }\end{array}$ & $\begin{array}{l}\text { Merulius tremellosus Schrad. (T, BP, } \\
\text { pod. 1-2) }\end{array}$ & $\begin{array}{l}\text { Chlorociboria aeruginascens } \\
\text { (Oeder) Seaver (T-To, BP-BS, } \\
\text { podr.1-4) }\end{array}$ \\
\hline $\begin{array}{l}\text { Ceriporiopsis costaricensis } \mathrm{M} \text {. Mata } \\
\text { \& Ryvarden (T, BP, podr. 1-3) }\end{array}$ & Omphalina sp.1 (T, BP, podr. 1-3) & $\begin{array}{l}\text { Daldinia concentrica (Botton) } \\
\text { Ces. \& De Not (T, BP, podr. 1) }\end{array}$ \\
\hline $\begin{array}{l}\text { Crepidotus sp. } 1 \\
\text { (T-To, BP-BS, podr. 1-3) }\end{array}$ & $\begin{array}{l}\text { Perenniporia sp. } 1 \\
(\mathrm{~T}, \mathrm{BP}, \text { podr. } 1-3)\end{array}$ & $\begin{array}{l}\text { Dasyscyphus sp. } 1 \\
\text { (To, BS, podr. 1) }\end{array}$ \\
\hline $\begin{array}{l}\text { Cyptotrama sp.1 } \\
\text { (T, BP, podr.1-2) }\end{array}$ & $\begin{array}{l}\text { Phellinus cf. fastuosus } \\
\text { (To, BS, podr. 1) }\end{array}$ & $\begin{array}{l}\text { Hymenoscyphus sp.1 } \\
\text { (T-To, BP-BS, podr. 1-2) }\end{array}$ \\
\hline $\begin{array}{l}\text { Dictyopanus pusillus (Pers. ex Lév.) } \\
\text { Singer (T, BP, podr.1) }\end{array}$ & $\begin{array}{l}\text { Phellinus sarcites (Fr.) Ryvarden (T, } \\
\text { BP, podr. 1) }\end{array}$ & $\begin{array}{l}\text { Hypoxylon bovei var. } \\
\text { microsporum } \\
(\mathrm{T}, \mathrm{BP}, \text { podr.1-4) }\end{array}$ \\
\hline $\begin{array}{l}\text { Fistulina hepatica (Schaeff.) With. } \\
\text { (T-To, BP-BS, podr.1 ) }\end{array}$ & $\begin{array}{l}\text { Pleurotus djamor (Rumph:Fr.) } \\
\text { Boedijn (T, BP, podr.2) }\end{array}$ & $\begin{array}{l}\text { Hypoxylon lienhwacheense } \\
\text { Y.M. Ju \& J.D. Rogers } \\
\text { (T, BP, podr. 1) }\end{array}$ \\
\hline $\begin{array}{l}\text { Galerina sp.1 } \\
\text { (T-To, BP-BS, podr. 1-2) }\end{array}$ & $\begin{array}{l}\text { Pluteus cervinus (Schaeff.) P. Kumm. } \\
\text { (T, BP-BS, podr. 1-2) }\end{array}$ & $\begin{array}{l}\text { Lachnum sp.1 } \\
\text { (T-To, BP-BS, podr. 1-3) }\end{array}$ \\
\hline $\begin{array}{l}\text { Ganoderma applanatum (Pers.) Pat. } \\
\text { (T-To, BP-BS, podr. 1-4) }\end{array}$ & $\begin{array}{l}\text { Psathyrella sp. } \\
\text { (T-To, BP-BS, podr. 1-3) }\end{array}$ & $\begin{array}{l}\text { Orbilia sp.1 } \\
\text { (T-To, BP-BS, podr.1-3) }\end{array}$ \\
\hline $\begin{array}{l}\text { Ganoderma cf. lucidum } \\
\text { (T, BP, podr. 2-4) }\end{array}$ & $\begin{array}{l}\text { Ramaria sp.1 } \\
(\mathrm{T}, \mathrm{BP}, \text { podr. } 1-4)\end{array}$ & $\begin{array}{l}\text { Xylaria adscendens (Fr.) Fr. (T, } \\
\text { BS, podr.2) }\end{array}$ \\
\hline $\begin{array}{l}\text { Gymnopilus sp.1 } \\
\text { (T-To, BP-BS, podr. 1-2) }\end{array}$ & $\begin{array}{l}\text { Schizopora paradoxa (Schrad.) Donk } \\
\text { (T, BS, podr. 1-3) }\end{array}$ & $\begin{array}{l}\text { Xylaria badia Pat. (To,BS, } \\
\text { podr. 2-3) }\end{array}$ \\
\hline $\begin{array}{l}\text { Gymnopus sp. } 2 \\
\text { (T-To, BP-BS, podr. 1-3) }\end{array}$ & $\begin{array}{l}\text { Stereum ostrea (Blume \& T. Nees) Fr. } \\
\text { (T, BP, podr. } 1-3)\end{array}$ & $\begin{array}{l}\text { Xylaria aff. brevipes } \\
\text { (To, BS, podr. 2) }\end{array}$ \\
\hline $\begin{array}{l}\text { Hygrocybe cantharellus (Fr.) Murrill } \\
(\mathrm{T}, \mathrm{BP}, \text { podr. } 1)\end{array}$ & $\begin{array}{l}\text { Stereum hirsutum (Willd.) Pers. (T, } \\
\text { BP, podr. 1-4) }\end{array}$ & $\begin{array}{l}\text { Xylaria cf. coccophora } \\
\text { (T, BP, podr. } 1)\end{array}$ \\
\hline $\begin{array}{l}\text { Hygrocybe laeta (Pers.) P. Kumm. } \\
\text { (T, BP, podr. 1-2) }\end{array}$ & Trametes sp.1 (T, BP, podr. 1-3) & $\begin{array}{l}\text { Xylaria cf. consociata } \\
(\mathrm{T}, \mathrm{BP}, \text { podr. } 1-2)\end{array}$ \\
\hline $\begin{array}{l}\text { Hymenochaete rubiginosa (Dicks.) } \\
\text { Lév. (T-To, BP-BS, podr. 1-4) }\end{array}$ & $\begin{array}{l}\text { Trechispora microspora (P.Karst.) } \\
\text { Liberta (To, BS, podr. } 1-3)\end{array}$ & $\begin{array}{l}\text { Xylaria cubensis (Mont.) Fr. } \\
\text { (To, BS, podr. 2) }\end{array}$ \\
\hline $\begin{array}{l}\text { Hypholoma capnoides (Fr.) P. Kumm. } \\
\text { (T-To, BP-BS, podr. 1-2) }\end{array}$ & $\begin{array}{l}\text { Tremella fuciformis Berk. (T, BP-BS, } \\
\text { podr. 1) }\end{array}$ & $\begin{array}{l}\text { Xylaria cf. filiformis (T, BS, } \\
\text { podr. 1) }\end{array}$ \\
\hline $\begin{array}{l}\text { Hypholoma subviride (Berk. \& Curtis) } \\
\text { M.A. Dennis (T, BP-BS, podr. 1-2) }\end{array}$ & $\begin{array}{l}\text { Tremella mesenterica Retz. (T, BS, } \\
\text { podr. 1) }\end{array}$ & \\
\hline $\begin{array}{l}\text { Inonotus fimbriatus L.D. Gómez \& } \\
\text { Ryvarden (T, BS, podr.1) }\end{array}$ & $\begin{array}{l}\text { Trichaptum biforme (Fr.) Ryvarden } \\
(\mathrm{T}, \mathrm{BP}, \text { podr. } 1-2)\end{array}$ & \\
\hline $\begin{array}{l}\text { Ischnoderma resinosum (Schrad.) P. } \\
\text { Karst. (T, BP, podr. 1-3) }\end{array}$ & $\begin{array}{l}\text { Xeromphalina sp.1 (T, BP-BS, podr. } \\
1-4)\end{array}$ & \\
\hline $\begin{array}{l}\text { Laetiporus sulphureus (Bull.) Murrill } \\
\text { (T-To, BP-BS, podr. 1-2) }\end{array}$ & $\begin{array}{l}\text { Xylobolus subpileatus (Berk. \& M.A. } \\
\text { Curtis) Boidin (T-To, BP-BS, podr. 1- } \\
\text { 4) }\end{array}$ & \\
\hline
\end{tabular}

*Los niveles de podredumbre presentes en los troncos o tocones donde se encontraban los hongos se indican como podr. 1-4. 
La mayoría de las especies descomponedoras de madera observadas se encontraron en troncos o tocones con nivel de podredumbre 1 (53 especies, 89\%); sin embargo, su número disminuyó conforme avanzó el nivel de podredumbre, ya que sólo se recolectaron 23 especies $(38 \%)$ en troncos o tocones con niveles 3-4 de podredumbre, incluidas en algunos casos especies diferentes a $X$. subpileatus o H. rubiginosa (cuadro1).

Algunos troncos presentaban partes que no se encontraban en contacto directo con el suelo, en estos casos, se observó niveles de podredumbre más bajos que los que estaban completamente a nivel del suelo.

La podredumbre más común fue la blanca en bolsas (fig. 4), en unos pocos se observó podredumbre blanca uniforme y en otra podredumbre café.

\section{Número de especies vs. tamaño del tronco}

El número de especies observadas en cada tronco en el bosque primario, así como en troncos o tocones en el secundario fue muy variable. Algunos troncos del bosque primario tenían desde seis hasta 39 especies diferentes, y en el bosque secundario se identificaron desde cinco hasta 22 especies diferentes en troncos o tocones, durante los cinco años de estudio. Aunque el número de especies fue igual en algunos troncos y tocones, no siempre se observaron las mismas especies. Al analizar la relación entre la circunferencia de los troncos en ambos bosques y el número de especies observadas de hongos por medio de regresión lineal, se encontró una muy pequeña tendencia a bajar el número de especies con respecto al tamaño del tronco; sin embargo, se considera necesario un mayor número de troncos para confirmar con certeza esta tendencia.

\section{Variaciones en la humedad del suelo y de las maderas}

Las precipitaciones más altas en la zona se dieron durante los años 2008 y 2010, y las más bajas en el 2009, 2011 y 2012 (fig. 2). Los porcentajes de humedad en los troncos y suelos del bosque primario y secundario variaron en los diferentes meses muestreados de acuerdo a las condiciones climatológicas. En el caso de las maderas, los porcentajes fueron casi siempre mayores de $60 \%$, excepto en los meses de febrero (2010 y 2012), marzo (2012) y abril (2011), donde se presentaron los más bajos (19-33\%). La retención de humedad en los troncos se vio favorecida por la cubierta gruesa de musgos y la gran cantidad de hojarasca sobre ellos. La humedad del suelo siempre se mantuvo alta $(>50 \%)$, aún en periodos de poca precipitación posiblemente debido a la capa gruesa de hojarasca presente casi todo el tiempo.

\section{Cámaras de podredumbre}

\section{Crecimiento de Hymenochaete rubiginosa en los bloques de madera}

A los pocos días de colocados los bloques en las cámaras, se observó un crecimiento micelial rápido a los lados de la madera. A los seis meses, el micelio los había cubierto completamente y formaba una masa compacta abultada de apariencia aterciopelada de color pardo rojizoamarillento (fig. 4).

\section{Crecimiento de Xylobolus subpileatus en los bloques de madera}

A los pocos días de colocados los bloques en las cámaras, se observó crecimiento micelial a los lados de la madera. A los 20 días, ya estaban todos cubiertos por un micelio amarillento (fig. 4) de textura farinácea que luego se compactó y oscureció a un tono amarillo parduzco a los cuatro meses. Al observar el micelio al microscopio se visualizaron las hifas y los cistidios con cristales típicos de la especie. No se observaron estructuras reproductoras. 


\section{Crecimiento de Trametes versicolor en los bloques de madera}

Después de dos semanas se observó crecimiento micelial sobre los bloques de madera. A los dos meses se observó un micelio postrado sobre la parte superior del bloque y más algodonoso y compacto a los lados y extremos con leves cambios de color en algunas zonas. A los cuatro meses había formado una capa densa de color blanco con zonas pardo rojizas a pardo negruzcas sobre los bloques de madera, y en las paredes y tapas de los frascos (fig. 4).

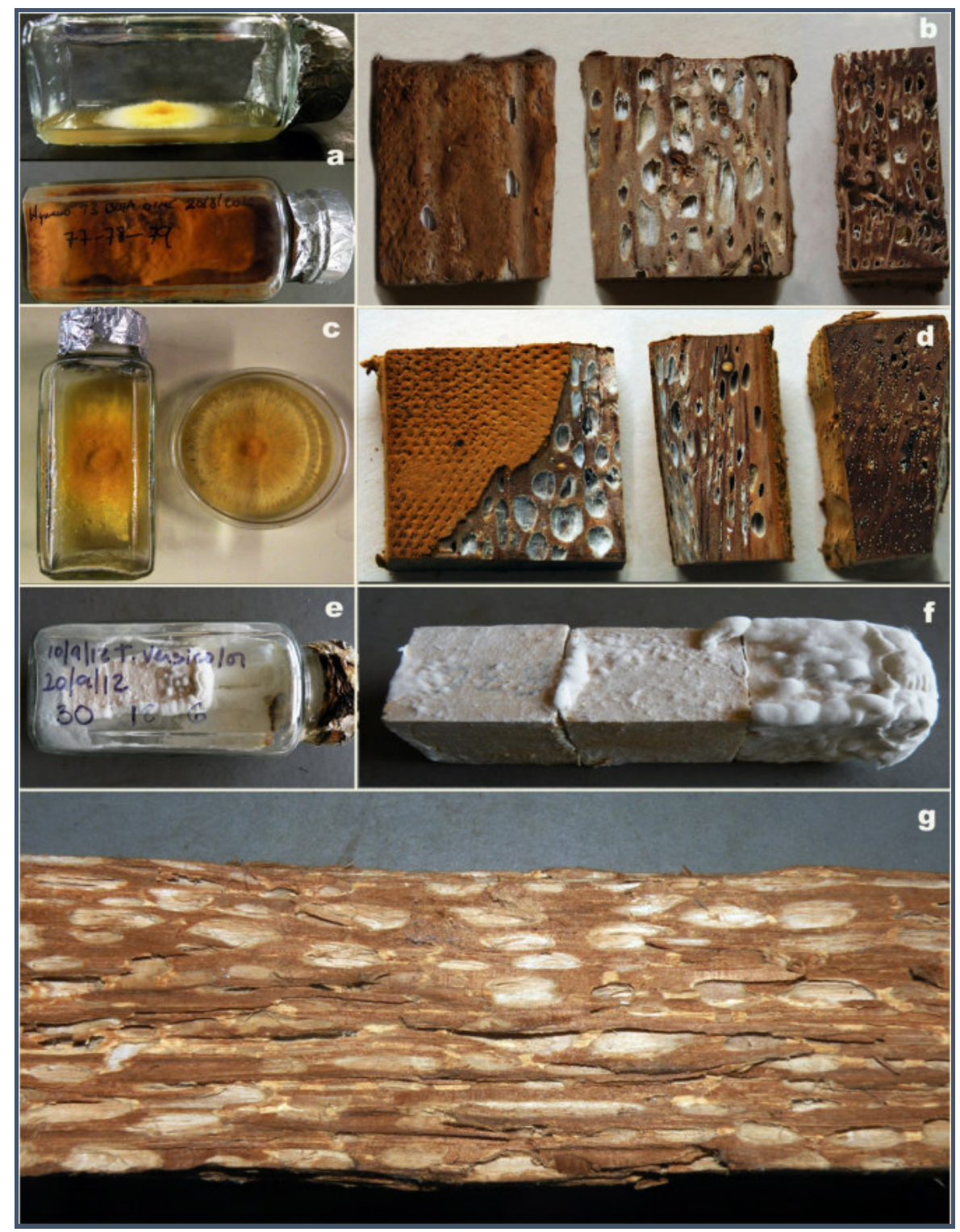

Fig. 4. Cámaras de podredumbre y muestra de madera de roble recolectada en el campo. a. Micelio y bloques de madera después de ocho meses de exposición a Hymenochaete rubiginosa; b. Podredumbre blanca en bolsas en bloques de madera expuestos a H. rubiginosa (ocho meses); c. Micelio de Xylobolus subpileatus; d. Podredumbre blanca en bolsas en bloques expuestos a Xylobolus subpileatus (ocho meses); e y f. Bloques expuestos a Trametes versicolor (cuatro meses); g. Típica podredumbre blanca en bolsas. 


\section{Pérdidas de peso}

Los resultados del análisis estadístico de los datos de las pérdidas de peso de las maderas expuestas a $H$. rubiginosa y $X$. subpileatus, se muestran en el cuadro 2.

Se encontró una gran variación en la pérdida de peso de los bloques de madera utilizados en todas las cámaras. Los porcentajes variaron desde $2.08-51.06 \%$ a los ocho meses y tres semanas de exposición. Sin embargo, los promedios de pérdidas de peso de las maderas expuestas a ambos hongos fueron semejantes (cuadro 2).

Los porcentajes de pérdidas de peso de los bloques de madera expuestos a $T$. versicolor mostraron también una gran variación (4.16-44.44\%) con un promedio de 24.16 y una desviación estándar de 10.47; este resultado se obtuvo en bloques expuestos al hongo durante cuatro meses, por lo que no se analizaron en conjunto con los de los otros dos hongos.

Cuadro 2. Comparación de las pérdidas de peso (g) de los bloques de maderas expuestos a los dos hongos seleccionados y los testigos durante ocho meses y tres semanas.

\begin{tabular}{|l|c|c|c|}
\hline Hongo & Hymenochaete rubiginosa & Xylobolus subpileatus & Testigos \\
\hline Promedio & 23.27 & 22.12 & 3.24 \\
\hline Desviación estándar & 10.91 & 8.96 & 2.87 \\
\hline
\end{tabular}

Observación de cortes de bloques de madera al microscopio de luz

\section{Maderas expuestas a Xylobolus subpileatus e Hymenochaete rubiginosa}

Al cortar los bloques de madera por la mitad se observó para ambos tratamientos la típica podredumbre blanca en bolsas comunicada para las especies de estos géneros (Otjen \& Blanchette, 1984) (fig. 4b y d). A la hora de realizar los cortes con el micrótomo de deslizamiento, estos se rompían con facilidad, lo cual no permitió que se tomaran buenas fotografías. Sin embargo, se logró observar hifas en las células del parénquima del xilema secundario. En las secciones transversales, se observó hifas principalmente sobre las células del parénquima axial paratraqueal y en las paredes secundarias de los elementos de los vasos (elementos conductores).

Dado que la descomposición causada por Trametes versicolor ha sido ampliamente estudiada, no se incluyó el estudio anatómico de cortes procedentes de bloques de madera expuestos a este hongo.

\section{Análisis químicos:}

Al comparar la composición química de los bloques de maderas expuestos a $X$. subpileatus e $H$. rubiginosa con la de los bloques testigo, se observaron algunas diferencias en los porcentajes de los compuestos analizados. Los bloques de madera expuestos a X. subpileatus presentaron los porcentajes más altos de lignina, extractos totales e inorgánicos, y los más bajos de holocelulosa; los bloques expuestos a $H$. rubiginosa presentaron los porcentajes más bajos de lignina, pero al igual que $X$. subpileatus, los más altos de extractos totales e inorgánicos; sin embargo, los porcentajes de holocelulosa fueron similares a los de los bloques testigo (cuadro 3). 
Cuadro 3. Composición de los bloques de madera de roble testigos y expuestos en cámaras de podredumbre a dos hongos seleccionados después de ocho meses de exposición*.

\begin{tabular}{|l|c|c|c|c|c|c|}
\hline Compuesto & Patrón & $\begin{array}{c}\text { Coeficiente } \\
\text { de variación } \\
(\%)\end{array}$ & $\begin{array}{c}\text { Xylobolus } \\
\text { subpileatus }\end{array}$ & $\begin{array}{c}\text { Coeficiente de } \\
\text { variación } \\
(\%)\end{array}$ & $\begin{array}{c}\text { Hymenochaete } \\
\text { rubiginosa }\end{array}$ & $\begin{array}{c}\text { Coeficiente de } \\
\text { variación } \\
(\%)\end{array}$ \\
\hline Holocelulosa & 75.75 & - & 73.06 & - & 75.60 & - \\
\hline Lignina & 18.42 & 0.11 & 18.73 & 2.22 & 16.10 & 1.51 \\
\hline $\begin{array}{l}\text { Extractos } \\
\text { totales }\end{array}$ & 5.45 & 1.08 & 7.26 & 0.11 & 7.58 & 1.03 \\
\hline Inorgánicos & 0.38 & 0.63 & 0.98 & 1.74 & 0.72 & 1.78 \\
\hline
\end{tabular}

*Todos los porcentajes están dados en base seca.

\section{DISCUSIÓN}

Varios estudios han comunicado una gran heterogeneidad presente en troncos individuales durante el proceso de descomposición en bosques de zonas templadas, sobre todo en el nivel de podredumbre 3. Esta heterogeneidad se ha relacionado con la variabilidad en las tasas de descomposición, posiblemente debidas a la estructura interna de la madera, a los diferentes grupos de hongos que la degradan, a las diferencias en actividades enzimáticas y a influencias microclimáticas (Grove et al., 2011; Pyle \& Brown, 1999; Větrovský et al., 2011).

Los resultados de este estudio coinciden con estos hallazgos ya que se presentó una gran variabilidad en los niveles de podredumbre en un mismo tronco o tocón; lo cual podría estar relacionado con la variedad de especies de hongos descomponedores de madera presentes en estos sustratos, con las características químicas de la madera de roble que le confieren alta resistencia a la descomposición (Highley, 1995), y con las condiciones microclimáticas de los bosques, los cuales presentaron variaciones en temperatura y humedad a través del año. A pesar de esta variabilidad, el mayor porcentaje de madera se mantuvo durante los cinco años de estudio, en la categoría de nivel 1 de podredumbre, y solo en algunos troncos o tocones estaba la corteza suelta o la habían perdido. Aquellos que presentaron partes con niveles 3 de podredumbre, la albura ya estaba bastante deshecha, pero permanecía una buena parte del duramen. En los casos de los niveles 4, que se presentaron en partes muy limitadas de los troncos, el duramen ya se encontraba bastante descompuesto.

Kubartová et al. (2012) encontraron en su estudio de comunidades fúngicas en troncos que los descomponedores de madera eran más abundantes en troncos menos descompuestos. Resultados semejantes se observaron en este estudio donde el número de especies causantes de podredumbre disminuyó conforme avanzó la podredumbre, esto ha sido relacionado con cambios en los componentes de la madera al avanzar el proceso de descomposición y la entrada de hongos más generalistas o especialistas al final del proceso (Iršènaitė \& Kutorga, 2006; Kubartová et al., 2012; Nordén \& Paltto, 2001, Taudiere, y otros, 2017).

Grove et al. (2011) han argumentado que el sistema de clasificación de descomposición en diferentes niveles como el utilizado en este estudio, sirve para capturar la trayectoria de la pérdida de biomasa, pero que no caracteriza la complejidad ecológica del proceso que da origen a esta pérdida. Por lo tanto, recomiendan partir los troncos y ubicarlos en clases de diámetros que reflejen diferencias en la madurez de los árboles que les dieron origen, lo cual permite agregar datos de descomposición a través de todo el estudio, lo que da un sentido más ecológico. A pesar de no haberse utilizado esta metodología en el presente estudio, se considera que los resultados obtenidos son valiosos ya que dan una idea del proceso de descomposición de los troncos y tocones de roble en bosques montanos neotropicales, de los macrohongos involucrados y del posible lapso de tiempo que tomaría la total descomposición de esta madera 
bajo las condiciones imperantes en la zona. Dicha información puede ser utilizada en futuras investigaciones que involucren procesos de descomposición en bosques de altura en zonas tropicales.

Varias especies de Xylobolus e Hymenochaete han sido citadas comúnmente en roble (Karami et al., 2014; Mirić, 2005; Otjen \& Blanchette, 1984; Taudiere, y otros, 2017). Carranza et al. (2018) comunicaron la amplia distribución $X$. subpileatus e H. rubiginosa en la mayoría de los troncos y tocones del bosque primario y secundario en Dota. Esto da una indicación de su gran adaptabilidad a las condiciones de estos bosques y a la utilización del roble como sustrato, lo cual se confirmó al observar los resultados de las pérdidas de peso causadas por los dos hongos en las pruebas de laboratorio.

Nordén \& Paltto (2001) han mencionado un aumento en la riqueza de otras especies de hongos pertenecientes al grupo de corticeáceos s.l. conforme la madera alcanza altos porcentajes de descomposición. En el presente trabajo no se observó, al aumentar la podredumbre, un incremento en especies de este grupo en ninguno de los troncos o tocones en los bosques. La mayoría de los troncos y tocones sólo tenían los cuerpos fructíferos de Xylobolus subpileatus e Hymenochaete rubiginosa, aún en las partes con niveles más avanzados de podredumbre.

Estudios llevados a cabo con Xylobolus frustulatus y con miembros del orden Hymenocheaetales han demostrado la capacidad de estos hongos de causar podredumbre blanca en troncos de roble y pino, respectivamente (Karami et al., 2014; Mirić, 2005; Otjen \& Blanchette, 1984; Taudiere, y otros, 2017). X. frustulatus es capaz de una deslignificación selectiva lo que causa pérdidas de peso de hasta el $97 \%$, seguido por la remoción de xilosa (96\%) y luego manosa (69\%) durante el proceso incipiente de descomposición, lo que da como resultado la apariencia de podredumbre blanca en bolsas en la madera, compuesta de tejidos blancos celulósicos sin lignina (Otjen \& Blanchette, 1984).

De acuerdo con las observaciones microscópicas de los cortes de los bloques expuestos a $X$. subpileatus e $H$. rubiginosa, el ataque de los hongos a la madera se inició en el parénquima radial (rico en reservas nutritivas), lo cual facilitó el desplazamiento del micelio a lo interno de la madera, por sus conexiones con el parénquima axial, con los elementos conductores de agua y minerales (elementos de los vasos) y con los elementos estructurales (fibras libriformes). Una vez avanzada la invasión y digestión del parénquima paratraqueal en una acción centrípeta, los hongos actuaron sobre las paredes secundarias de los elementos de los vasos (elementos conductores), lo que dio lugar a una descomposición completa de sus paredes, que generó como resultado un arreglo laxo de los tejidos, y la aparición de "bolsas" o "aperturas" grandes dentro de la madera. Estas observaciones coinciden con lo comunicado por Otjen \& Blanchette (1984).

Pruebas de podredumbre con bloques de madera de roble expuestas a Trametes versicolor, han dado como resultado pérdidas de peso que oscilan de $1-83.35 \%$ durante diferentes periodos de exposición (3-6 meses), pero con un patrón de podredumbre blanca uniforme, con apariencia esponjosa o fibrosa (Guilley et al., 2004; Szewczyk \& Molińska-Glura, 2010), muy diferente a la observada con $X$. subpileatus e H. rubiginosa. En este estudio el crecimiento de $T$. versicolor en los bloques de madera de roble fue muy rápido y agresivo, lo que se reflejó en los porcentajes de pérdida de peso a los cuatro meses semejantes a los obtenidos con X. subpileatus e H. rubiginosa en ocho meses.

Las pérdidas de peso in vitro fueron muy variables en los bloques expuestos a los tres hongos. Estas altas variaciones son comunes en este tipo de pruebas, tal como ha sido comunicado en otros estudios (Guilley et al., 2004; Sarmiento et al., 2016). Por lo general, se han atribuido a la presencia de diferentes zonas de albura y duramen en los bloques utilizados, con la subsecuente variación en la concentración de compuestos tóxicos, lo cual influye en los procesos de descomposición. 
Tanto en el campo como en las cámaras de podredumbre con X. subpileatus e H. rubiginosa, se observó el patrón de podredumbre blanca en bolsas, lo que indica que ambos hongos tienen la capacidad de degradar de forma similar tanto in vivo como in vitro estos componentes. Los resultados de los análisis químicos dan una idea de la diferencia en la forma o velocidad de utilización de los componentes principales de la madera (lignina y holocelulosas) por $X$. subpileatus e H. rubiginosa; sin embargo, se necesitan estudios más detallados para determinar con exactitud la preferencia por uno u otro componente durante la descomposición de la madera.

La mayoría de los hongos descomponedores de la madera recolectados en los troncos y tocones de roble han sido comunicados como causantes de podredumbre blanca excepto Laetiporus sulphureus y Fistulina hepatica que causan podredumbre café (Gilbertson \& Ryvarden, 1986, 1987).

La alta humedad observada tanto en las maderas como en los suelos de los bosques estudiados y las bajas temperaturas a través de casi todo el año, sumado a la alta durabilidad de la madera de roble, son aspectos muy importantes que se relacionan con la lenta descomposición de esta madera, ya que como se ha mencionado anteriormente, afectan los procesos metabólicos de los hongos (Pyle \& Brown, 1999).

Dada esta lentitud en los procesos de descomposición, es muy posible que la degradación total de la madera de roble en estos bosques montanos neotropicales dure varias décadas como han mencionado otros autores en zonas templadas (Freschet et al., 2012).

Conocer el diferente estado de podredumbre de los troncos y su tamaño es importante desde el punto de vista ecológico ya que esto da una idea de la diversidad de especies y de los procesos involucrados (Pyle \& Brown, 1999). Algunos trabajos han indicado una correlación entre las especies y el tamaño del sustrato; sin embargo, otros mencionan que diferencias en el tamaño de los troncos no tienen un efecto significativo sobre la comunidad (Kubartová et al., 2012; Taudiere, y otros, 2017). En el presente estudio fue difícil comprobar si el tamaño del tronco y las comunidades de hongos que en ellos se desarrollan tiene algún efecto en la descomposición de los troncos. Los bosques en los cuales se llevó a cabo el estudio son fragmentos pequeños, y en el caso del secundario ha sido intervenido hasta recientemente, por lo cual no se encontraron gran cantidad de troncos caidos para poder estudiarlos. Varias especies de macrohongos descomponedores de la madera fueron identificados tanto en el bosque primario como secundario, los cuales contribuyen en los procesos de descomposición de los troncos y tocones de roble junto con $X$. subpileatus e $H$. rubiginosa. Sin embargo, otras comunidades de hongos microscópicos que no fueron aislados o aquellos que no fructificaron durante la realización del estudio también contribuyen en estos procesos.

\section{CONCLUSIONES}

Los procesos de descomposición de la madera de roble en bosques montanos neotropicales siguen patrones semejantes a los observados en zonas templadas. Estos procesos parecen no variar en fragmentos de bosques primarios comparados con los de bosques en regeneración que se encuentren cercanos y que comparten condiciones climáticas y una micobiota semejante, sobretodo de especies descomponedoras de la madera.

Los niveles de descomposición presentes en un mismo tronco o tocón son muy variables, y su avance no es uniforme, por lo cual es difícil representarlo en los niveles de podredumbre comúnmente utilizados en este tipo de estudio, ya que puede existir mayor variabilidad de la que sugiere un nivel en particular. Por lo tanto, cada tronco presentará diferente composición de especies y contribuirá de una forma diferente en el funcionamiento ecológico. 
Xylobolus subpileatus e Hymenochaete rubiginosa son muy abundantes tanto en el bosque primario como en el secundario, y, por lo tanto, juegan un papel muy importante en la descomposición de los troncos y tocones en estos bosques.

Se puede considerar que las tasas de descomposición de la madera de roble en estos bosques montanos neotropicales de Costa Rica pueden ser semejantes a las comunicadas para esta madera en zonas templadas; por lo tanto, el aporte de carbono proveniente de estos sustratos al ecosistema es lento. Sin embargo, se recomiendan estudios más detallados para determinar con exactitud dichas tasas y la contribución de carbono de estos bosques comparado con los de zonas bajas tropicales.

La información generada en esta investigación contribuye al conocimiento de la descomposición de la madera de roble, y podrá ser utilizada para un manejo sustentable de los recursos que aún se encuentran en estos ambientes y monitorear cambios a través del tiempo.

\section{AGRADECIMIENTOS}

A Jocelyn Villeaux y Jorge Rojas propietarios de los bosques por permitirnos realizar esta investigación. A Jack D. Rogers y Rosario Medel por su ayuda en la identificación de Xylariales. A Héctor Espinoza por su colaboración en la caracterización de los bosques. A Paul Hanson por la revisión del resumen en inglés. A los estudiantes que nos ayudaron en la recolecta de muestras y trabajo de laboratorio. Al Centro de Servicios Estudios Básicos de Ingeniería-Hidrología, Instituto Costarricense de Electricidad por facilitarnos los datos de precipitación, temperatura y humedad relativa de las dos estaciones cercanas al sitio de estudio. A los revisores anónimos por sus acertados comentarios y sugerencias. La presente investigación fue financiada por la Vicerrectoría de Investigación, Universidad de Costa Rica (proyecto 731-A8-039).

\section{LITERATURA CITADA}

(ASTM), A. S. for T. and M. (Ed.). (2005). D2017-05 "Standard Test Method of Accelerated Laboratory Test of Natural Decay Resistance of Woods." Annual Book of ASTM Standards.

Boddy, L., \& Swift, M. J. (1984). Wood decomposition in an abandoned beech and oak coppiced woodland in SE England. Ecography, 7, 229-238.

Carpio Malavassi, I. M. (1992). Manual de procedimientos para la sección de Anatomía y Morfología de la madera INII-77-92. (L. de P. Forestales, Ed.). San Pedro: Editorial Universidad de Costa Rica.

Carranza, J., DiStefano, J. F., Marín, W., \& Mata, M. (2018). Estudio comparativo de los macrohongos presentes en troncos de roble en dos bosques montanos neotropicales de Costa Rica. Polibotanica, 45, 1-22.

Carranza, J., \& Ruiz-Boyer, A. (2001). Cultural studies of some genera of Basidiomycetes (Basidiomycota) from Costa Rica. Harvard Papers in Botany, 6(1), 57-84.

Chambers, J. Q., Higuchi, N., Schimel, J. P., Ferreira, L. V, \& Melack, J. M. (2000). Decomposition and carbon cycling of dead trees in tropical forests of the central Amazon. Oecologia, 122, 380-388.

Freschet, G. T., Weedon, J. T., Aerts, R., van Hal, J. R., \& Cornelissen, J. H. C. (2012). Interspecific differences in wood decay rates: insights from new short-term method to study long-term wood decomposition. Journal of Ecology, 100, 161-170. https://doi.org/10.1111/j.1365-2745.2011.01896.x

Gilbertson, R. L., \& Ryvarden, L. (1986). North American Polypores. Norway: Fungiflora.

Gilbertson, R. L., \& Ryvarden, L. (1987). North American Polypores. Norway: Fungiflora. 
Gomez, K., \& Gomez, A. A. (1984). Statistical Procedures for Agricultural Research (Second Edi). New York: John Wiley \& Sons.

Grove, S. J., Stamm, L., \& Wardlow, T. J. (2011). How well does a log decay-class system capture the ecology of decomposition?. A case-study from Tasmanian Eucalyptus oliqua forest. Forest Ecology and Management, 262, 692-700.

Guilley, E., Charpentier, J. P., Ayadi, N., Snakkers, G., Nepveu, G. B., \& Charrier, B. (2004). Decay resistance against Coriolus versicolor in Sessile oak (Quercus petrae Liebl.): analysis of the between-tree variability and correlations with extractives, tree growth and other basic wood properties. Wood Science \& Technology, 38, 539-554.

Highley, T. L. (1995). Comparative Durability of Untreated Wood in Use Above Ground. International Biodeterioration \& Biodegradation, 409-419.

Huhndorf, S. M., Lodge, D. J., Wang, C. J., \& Stokland, J. N. (2004). Macrofungi on woody substrata. In G. M. Mueller, G. F. Bills, \& M. S. Foster (Eds.), Biodiversity of Fungi. Inventory and Monitoring Methods (pp. 159-168). New York: Elsevier Academic Press.

Iršėnaitè, R., \& Kutorga, E. (2006). Diversity of fungi on decaying common oak coarse woody debris. Ekologija, 4, 22-30.

Karami, L., Fromm, J., Koch, G., Schmidt, O., \& Schmitt, U. (2014). Oak wood inhabiting fungi and their effect on lignin studied by UV microspectrophotometry. Maderas. Ciencia y Tecnologia, 16(2), 149-158.

Keller, M., Palace, M., Asner, G. P. Z., Pereira, J. R., \& Silva, J. N. M. (2004). Coarse woody debris in undisturbed and logged forests in the eastern Brazilian Amazon. Global Change Biology, 10, 784-795.

Kirk, T. K. (1973). The Chemistry and Biochemistry of decay. In D. D. Nicholas (Ed.), Wood Deterioration and its prevention by Preservative Treatments (1st ed., pp. 149-181). Syracuse: Syracuse University Press.

Kubartová, A., Ottosson, E., Dahlberg, A., \& Stenlid, J. (2012). Patterns of fungal communities among and within decaying logs, revealed by 454 sequencing. Molecular Ecology, 1-19.

Lindblad, I. (2001). Diversity and distribution of wood inhabiting Basidiomycetes on decomposing logs, examples from tropical and boreal forests. Oslo: University of Oslo.

Lisiewska, M. (1992). Macrofungi on special substrates. In H. Lieth (Ed.), Handbook of vegetation science (pp. 151-182). London: Kluwer.

Lodge, D. J. (1993). Nutrient cycling by fungi in wet tropical forests. In S. Isaac, J. C. Frankland, R. Watling, \& A. J. S. Whalley (Eds.), Aspects of Tropical Mycology (pp. 37-57). Cambridge.

Mirić, M. (2005). Decay of oak wood provoked by the fungus Stereum hirsutum (Wild.:Fr.) S.F.Gray. and its essential physiological requirements. Biblid, 9, 179-192.

Mueller, G. M., Bills, G. F., \& Foster, M. S. (2004). Biodiversity of Fungi. Inventory and Monitoring Methods. (G. M. Mueller, G. F. Bills, \& M. S. Foster, Eds.). New York.

Mueller, G. M., Halling, R. E., Carranza, J., Mata, M., \& Schmit, J. P. (2006). Saprotrophic and Ectomycorrhizal Macrofungi of Costa Rican Oak Forest. In M. Kappelle (Ed.), Ecology and Conservation of Neotropical Montane Oak Forests (pp. 55-68). Berlin: Springer-Verlag.

Muñoz Rivera, A. (2009). Efecto de la alteración del bosque sobre la estructura de ensamblajes de especies de macrohongos en San Gerardo de Dota. San Jose: Universidad de Costa Rica.

Nordén, B., \& Paltto, H. (2001). Wood-decay fungi in hazel wood: species richness correlated to stand age and dead wood features. Biological Conservation, 101, 1-8.

Ostrogović, M. Z., Marjanović, H., Balenović, I., Sever, K., \& Jazbec, A. (2015). Decomposition of fine woody debris from main tree species in lowland oak forest. Polish Journal of Ecology, 63, 247-259.

Otjen, L., \& Blanchette, R. A. (1984). Xylobolus frustulatus Decay of Oak: Patterns o Selective Delignification and Subsequent Cellulose Removal. Applied Environmental Microbiology, 47, 670-676. 
Recibido: 15/noviembre/2017

Aceptado: 27/septiembre/2018
Pyle, C., \& Brown, M. M. (1999). Heterogeneity of wood decay classes within hardwood log. Forest Ecology and Management, 114, 253-259.

Rodríguez Barreal, J. (1998). Patologia de la Madera. Madrid: Ediciones Mundi-Prensa.

Ruzin, S. E. (1999). Plant microtechnique and Microscopy. Oxford: Oxford University Press.

Sánchez Chacón, E., Carpio Malavassi, I. M., \& Arroyo, O. (1994). Ultraestructura de la madera de seis especies arborescentes de importancia comercial en Costa Rica. Revista de Biología Tropical, 42(2), 59-64.

Sarmiento, E., Carranza, J., \& Marín, W. (2016). In vitro wood decay of teak (Tectona grandis) by Rigidoporus cfr. microporus (Meripiliaceae, Polyporales, Basidiomycota). Polibotanica, 41, 67-78.

Scheffer, T. C. (1973). Microbial Degradation and the Casual Organisms. In D. D. Nicholas (Ed.), Wood Deterioration and its prevention by Preservative Treatments (First Edit, pp. 31-106). Syracuse: Syracuse University Press.

Schowalter, T. D., Caldwell, B. A., Carpenter, S. E., Griffiths, R. P., Harmon, M. E., Ingham, E. R., ... Moldenke, A. R. (1992). Decomposition of Fallen Trees: Effects of Initial Conditions and Heterotroph Colonization Rates. Tropical Ecosystems: Ecology and Management, 373-383.

Schowalter, T. D., Zhang, Y. L., \& Sabin, T. E. (1998). Decomposition and nutrient dynamics of oak Quercus spp logs after five years of decomposition. Ecography, 21, 3-10.

Szewczyk, W., \& Molińska-Glura, M. (2010). Decay of oak heartwood by selected fungi species. Acta Sci.Pol. Silv. Colendar.Rat. Ind. Lignar., 9(3-4), 29-36.

Taudiere, A., Bellanger, J. M., Moreau, P. A., Carcaillet, C., Christophe, A., Læssøe, T., . . . Richard, F. (2017). Xylobolus subpileatus, a specialized basidiomycete functionally linked to old canopy gaps. Canadian Journal of Forest Research, 47(7), 965-973.

Větrovský, T., Voříšková, J., Šnajdr, J., Gabriel, J., \& Baldrian, P. (2011). Ecology of coarse wood decomposition by the saprotrophic fungus Fomes fomentarius. Biodegradation, 22, 709-718.

Whalley, A. J. S. (1993). Tropical Xylariaceae: their distribution and ecological characteristics. In S. Isaac, J. C. Frankland, R. Watling, \& A. J. S. Whalley (Eds.), Aspects of Tropical Mycology (pp. 103-119). Cambridge. 